\title{
Effect of Number of Electrodes on Electrical Performance of Surface Dielectric Barrier Discharge Plasma Actuator
}

\author{
Andrew Ragai Henry Rigit \\ Department of Mechanical and \\ Manufacturing Engineering, \\ Universiti Malaysia Sarawak \\ Kota Samarahan, Malaysia \\ arigit@unimas.my \\ Chi Hung Chong \\ Department of Mechanical and \\ Manufacturing Engineering, \\ Universiti Malaysia Sarawak \\ Kota Samarahan, Malaysia \\ chongchihung1407@hotmail.com
}

\author{
Imran Ali \\ Department of Electrical and Electronic \\ Engineering \\ Universiti Malaysia Sarawak \\ Kota Samarahan, Malaysia \\ imran.ikmom@gmail.com
}

\author{
Tiaw C Boon \\ Department of Mechanical and \\ Manufacturing Engineering, \\ Universiti Malaysia Sarawak \\ Kota Samarahan, Malaysia \\ tiaw_cb@yahoo.com
}

\begin{abstract}
Dielectric barrier discharge (DBD) represent their wide application in controlling aerodynamic flow by plasma actuators. Their effectiveness is affected by the shape, type size, and thickness of electrodes. This paper investigates the influence of the number of electrodes on the electrical functioning of surface DBD plasma actuator. For this purpose, five different configurations of plasma actuator with varying electrodes number from 4 to 8 are tested. The gap between the electrodes and the length and width of each electrode remains constant in all these configurations. It was found that on increasing the number of electrodes and the applied frequency $(1 \mathrm{kHz}$ to $5 \mathrm{kHz})$ the value of maximum withstand voltage was decreased. However, the discharge power was increasing slightly on increasing the number of electrodes. This slight change in discharge power resulted in the significant plasma formation on the surface of the plasma actuator, the effect was visually captured by a CCD camera.
\end{abstract}

Keywords—plasma actuator, surface dielectric barrier discharge, plasma formation,

\section{INTRODUCTION}

Controlling the motion of fluids has been a pioneering challenge for humans for decades. Fluid flow can be controlled following the active method to improve the aerodynamic and hydrodynamic performances like increasing the lift and reducing the drag or improving the power required in pumping the fluids through pipes. The active flow method can be followed by bringing a mechanical change in the shape of an object (i.e moving leading and tail edges of the airfoil) to control the fluid flow [1][2]. However, controlling the flow mechanically leads to some intrinsic drawbacks like a significant increase in the weight, complicated components, and a lack of instant response. On the other hand, flow controlled by plasma actuators, utilizing the electrohydrodynamic (EHD) forces, has been considered as a promising active flow controlling device for the next generation [3]. The plasma actuator is an electrical device that usually has two electrodes, whereas, they can be arranged by either encapsulated or non-encapsulated configuration. A dielectric material used in actuators is made up of ceramics material, Kapton, quartz, or glass [4]. Its function in the plasma actuator is to separate the electrodes and the actuator, the plasma actuator is then called Surface Dielectric Barrier Discharge (SDBD) plasma actuator.
The plasma can be discharged by the SDBD plasma actuators when a dielectric material is added in the discharge regime [5], and this can be utilized in controlling airflow on the aerodynamic geometry [6]. The purplish observed plasma is actually an ionized air that consists of charged particles (ions and electrons) most of which appear electrically neutral and have the ability to generate the steady jet within seconds [7]. The plasma actuators show benefits in having a negligible weight, easiness in repairing, and the flexibility to install on the curved surface of the airfoil, and this makes them play a vital role in the aviation industry. Furthermore, the performance of the SDBD plasma actuator is dependent on the size, shape, thickness, and type of dielectric material and electrodes and the gap between them. To this end, some of the researchers [8]-[10] replaced the plate-shaped electrode with a wire-shaped and found that the effectiveness of the actuator was increased, in view of EHD force and stream-free flow production. The topology of ionic wind can be intensely modified when the edge of an active electrode is not straight [11]. An investigation carried out by [12] highlighted that the morphology of plasma, the velocity of ionic wind, and the discharge current are different according to the shape of the air-exposed active electrode. Apart from this, they also concluded that the applied high voltages resulted in the highest recorded ionic wind velocity production with a plate and sawtooth-shaped active electrode. However, it was not tested to observe what will be the effect when the number of active electrodes is increased.

In the present paper, the performance of the SDBD plasma actuator is investigated with a different number of air-exposed active electrodes. The independent variables are the applied frequency, withstand voltages, and the number of electrodes, whereas the thickness of the electrode is set as a constant variable. The optimizing factor used to observe the performance is the number of electrodes. The effectiveness of the SDBD plasma actuator was investigated in terms of maximum withstand voltages and discharge power with 4,5 , 6,7 , and 8 number of active electrodes, respectively.

\section{EXPERIMENTAL SETUP}

The experimental work is divided into three sections. These include a high voltage generating system, the plasma actuator, and the recording instruments. The formation of plasma will energize the particles in the air such as ions and 